\title{
Review of: "Hypoxia-induced HIF1 $\alpha$ activation regulates small extracellular vesicle release in human embryonic kidney cells"
}

\author{
Sushil Kumar ${ }^{1}$, Kurt Stenmark ${ }^{1}$, Cheng-Jun $\mathrm{Hu}^{1}$ \\ 1 University of Colorado Anschutz Medical Campus
}

Potential competing interests: The author(s) declared that no potential competing interests exist.

In their paper "Hypoxia-induced HIF1 $\alpha$ activation regulates small extracellular vesicle release in human embryonic kidney cells," Muñiz-García et al. seek to better understand the molecular mechanisms underlying increased extracellular vesicle (EV) release by cells in response to hypoxic stress. They chose six different cell types (3T3L1, NIH3T3, HEK293T, hUMSCs, MDA-MB231, and AC16) for initial screening of small EV (sEV) release under hypoxic compared to normoxic conditions. They found hypoxia enhanced sEV release in most of the cell lines. To address whether HIF1 or HIF2 activation is involved in this process, they used shRNA to knock-down HIF1 $\alpha$ or HIF2 $\alpha$ individually or in combination in HEK293T cells. They found that HIF1 $\alpha$, but not HIF2 $\alpha$, siRNA abolishes the effect of hypoxia on SEV release, suggesting HIF1 activation is necessary for increased SEV release in HEK293 cells in response to hypoxic stress.

Interestingly, when they stabilized HIF proteins in HEK293 cells grown in normoxic conditions, either by transfecting the cells with normoxia-stable versions of HIF1 $\alpha$ or HIF2 $\alpha$ expressing plasmids or treating the cells with a chemical inhibitor of the prolyl hydroxylase 2 (PHD2), they found no change in EV release, indicating that HIF activation is not sufficient for increasing EV release. Finally, the authors demonstrated that hypoxia activates autophagy and ER stress/unfolded protein response (UPR) in HEK293 cells. Thus, this study painted a picture that HIF1 activation is necessary, but not sufficient, for hypoxia-mediated increased EV release from cells and the potential involvement of autophagy and UPR in this process. This study strengthens the idea that hypoxia can enhance the number of sEVs released by cells in a cell type and hypoxia duration-dependent manner. However, the underlying mechanisms for hypoxia-induced EV release are still far from clear.

There are weaknesses in the experimental design that reduce the impact of the findings. The authors demonstrate that hypoxia enhanced SEV release in several cell lines in Figure 1 when normalizing sEV numbers by cellular lysate protein. Would this be true if the normalization was by cell number? In the cells studied, does hypoxia increase or decrease cell proliferation? The unwanted variation could be minimized by estimating SEV protein and then normalization with protein of cellular lysate. Alternatively, the SEV protein could be normalized to cell numbers similar to a paper published by Kumar et al. (2021). Examination of an exosome marker in SEVs was performed in HEK293T cells but it would be important to examine other cell lines. 
The expression of HIFla gene is universal, while HIF2a gene is only highly expressed in some specific cell types. Choosing HEK293T cells for the study concerning the role of HIF1 versus HIF2 in SEV release is probably not ideal, as HEK293T cells express deficient levels of HIF2 $\alpha$ (Hu et al., 2003). Future studies should include multiple cell types such as Hep3B cells in which both HIF1a and HIF2a genes are highly expressed, and HIF1 and HIF2 target genes are well established to address the relative role of HIF1 and HIF2 in the SEV release.

Notably, the authors found that HIF over-expression in normoxic HEK293 cells is insufficient to increase EV release, which prompted them to evaluate the role of other pathways, including autophagy and ER/UPR, in this process. As is frequently the case with novel studies, this study only reports the activation of these pathways, but not on the functional consequence related to EV release. Future experiments are needed to determine the effect of HIF in activating these pathways and what role these pathways play in the increased release of SEV. Cell lines from clear cell renal cell carcinoma, due to VHL mutation, exhibit consistent over-expression of HIFla and/or HIF2a protein. These cells might be a good platform to address if hypoxia-mediated increase of SEV release depends on activation of HIF or is due to HIF-independent activation of autophagy and ER/UPR pathways.

As the Authors stated in the Introduction, "The precise molecular mechanisms that contribute to enhanced EV formation and release during hypoxic conditions, however, remain unknown." Future studies should address if hypoxia increases SEV formation/biogenesis or releases, or both. Questions including whether hypoxia changes the composition of EVs and causes a shift in size distribution of released EVs should also be addressed.

\section{REFERENCES}

Muñiz-García A, Romero M, Falcón-Perez JM, Murray P, Zorzano A, Mora S. Hypoxia-induced HIF1 $\alpha$ activation regulates small extracellular vesicle release in human embryonic kidney cells. Sci Rep. 2022 Jan 27;12(1):1443.

Kumar S, Frid MG, Zhang H, et al. Complement-containing small extracellular vesicles from adventitial fibroblasts induce proinflammatory and metabolic reprogramming in macrophages. JCl Insight. 2021;6(21):e148382.

Hu CJ, Wang LY, Chodosh LA, Keith B, Simon MC. Differential roles of hypoxia-inducible factor lalpha (HIF1alpha) and HIF-2alpha in hypoxic gene regulation. Mol Cell Biol. 2003;23(24):9361-9374. 